\title{
Quality Of Life in Advanced Dementia
}

\section{Ladislav Volicer*}

School of Aging Studies, University of South Florida, Tampa FL, USA

*Corresponding author: Ladislav Volicer, School of Aging Studies, University of South Florida, Tampa FL, USA, E-mail: Ivolicer@usf.edu

Received date: July 22, 2016, Accepted date: July 24, 2016, Published date: August 01, 2016

Copyright: (c) 2016 Volicer L. This is an open-access article distributed under the terms of the Creative Commons Attribution License, which permits unrestricted use, distribution, and reproduction in any medium, provided the original author and source are credited.

\section{Editorial}

The aging of the world population is increasing the prevalence of people suffering from some form of a progressive degenerative disease, in most cases Alzheimer's disease. With no effective treatment that would cure or stop the progression of these diseases many people survive into an advanced stage of dementia. Optimal care for people with advanced dementia must not only include safe environment, adequate nutrition and personal hygiene, but should also promote high quality of life during the disease process. Quality of life is a subjective quality and is difficult to determine in people who cannot meaningfully communicate their feelings. However, a study investigating perceptions of quality of life among nursing home residents with different degrees of cognitive impairment, found that there were four key themes identified by the respondents: social contact, attachment, pleasurable activities, and affect [1].

People with advanced dementia (MiniMental State Examination (MMSE) score 1-9)[2] reported missing their families and not knowing anybody in the home, have not seen the home as their "home", and some of them said that they just visit or work there. When they were asked about organized activities, they answered "I've never heard of any. I don't know of any" and "Don't mind, don't do anything here", and when asked about their mood "Sometimes I wish I was dead cause there's nothing in life for me at all. I can't make anything out of life. I am unlucky with my family". These answers clearly indicate that there is a need for special attention to quality of life of people with advanced dementia. Unfortunately, this field is quite neglected and only $1.9 \%$ of studies investigating strategies for improvement quality of life in dementia considered advanced dementia [3].

Quality of life in people with dementia is difficult to evaluate objectively but several scales were developed for this purpose. However, their use in advance dementia is limited because several of them have a subjective component and floor effects. The Quality of Life in Alzheimer's Disease Scale [4] has been found useful only for persons with MMSE scores $\geq 3$. The Cornell-Brown Scale for Quality of Life in Dementia [5], derived from the Cornell Scale for Depression in Dementia [6] is limited by its requirement of a joint interview of caregiver and patients, and appears to be useful primarily with subjects having MMSE score $>8$. Use of the Quality of Life in Dementia scale (QUALIDEM) [7] is restricted by its requirement for subjective information from patients. The most useful for evaluation of quality of life in people with advanced dementia is the QUALID scale [8].

This review will point out the few strategies that were found to be effective in advanced dementia. Most of them did not measure their effect on quality of life but evaluated their effects on behavioral symptoms of dementia. It could be argued that presence of behavioral symptoms is stressful for people with dementia and, therefore, decreases their quality of life. However, some of the scales used (especially Cohen-Mansfield Agitation Inventory) [9]) do not consider apathy, which is very common in advanced dementia [10] and certainly decreases quality of life. Two comprehensive reviews related to this issue were recently published, one about different strategies for sensory stimulation [11] and the other for treatment of agitation $[12,13]$. Only strategies that are relevant for people with advanced dementia are included in Table 1.

Staff education: about dementia care was found to decrease behavioral symptoms of dementia in several studies. The education may take different forms, from Dementia Care Mapping to informal educational strategies [13]. Such an education is applicable to all stages of dementia and may change care strategies during the whole day and night seven days a week. However, even education using Dementia Care Mapping did not improve the quality of life of participants. The disadvantage of this strategy is, that it is time consuming and requires continuing education when new staff is hired.

Snoezelen: It is a mistake to consider all studies investigating the effect of Snoezelen as investigating the same strategy. The original description of the Snoezelen integrated program includes very intensive education program, getting detailed history and preferences by a family interview, doing stimulus preference screening, developing a plan and having $10 \mathrm{hrs}$ follow-up meetings [14]. That is very different from using just the multisensory stimulation equipment for a short period of time which was done in most follow-up studies. It is no surprise that the integrated program is effective in improving apathy, rebellious and aggressive behavior and symptoms of depression, while the short periods of multisensory stimulation alone do not have beneficial effects [13].

Music therapy: Music therapy is usually provided by a music therapist in several short sessions. There is good evidence that people with dementia enjoy these sessions but some programs require that participants are able to fill out a music evaluation form and follow directions. People with advanced dementia may not be able to do that. It is also not clear if the short occasional music sessions have long-term effect on behavioral symptoms of dementia and wellbeing [13].

Massage: Isolated episodes of massage do not have good effects on behavioral symptoms of dementia. In one study, agitation actually increased and the investigators postulate that this was caused by the unfamiliar research assistants and variations in usual activity [15]. This contrasts with beneficial effects of loving touch, which is a part of the Namaste Care program described below.

Namaste care: This program was not included in the recent reviews and, therefore, will be described in more detail. Namaste Care is a program specifically developed for people with advanced dementia and for others who cannot participate in usual activities [16]. A qualitative study using interviews with staff and family members reported that Namaste Care has two main principles: special environment and loving touch [17]. 
Page 2 of 3

\begin{tabular}{|l|l|l|l|l|l|l|l|l|l|}
\hline Strategy & $\begin{array}{l}\text { Duration of } \\
\text { strategy } \\
\text { /day }\end{array}$ & $\begin{array}{l}\text { Number } \\
\text { days/week }\end{array}$ & $\begin{array}{l}\text { Group } \\
\text { activity }\end{array}$ & $\begin{array}{l}\text { Environment } \\
\text { change }\end{array}$ & $\begin{array}{l}\text { Special } \\
\text { equipment }\end{array}$ & Supplies & Training & $\begin{array}{l}\text { Additional } \\
\text { staff }\end{array}$ & $\begin{array}{l}\text { Behavioral } \\
\text { effect }\end{array}$ \\
\hline $\begin{array}{l}\text { Staff } \\
\text { education [13] }\end{array}$ & 24 hrs & 7 & no & no & no & no & extensive & no \\
\hline $\begin{array}{l}\text { Snoezelen } \\
\text { Integrated } \\
\text { Program [11] }\end{array}$ & $24 \mathrm{hrs}$ & 7 & no & possibly & yes & no & extensive & no & yes \\
\hline $\begin{array}{l}\text { Snoezelen } \\
\text { episodes [11] }\end{array}$ & $30-40$ min & $2-3$ & no & yes & yes & no & no & no \\
\hline $\begin{array}{l}\text { Music therapy } \\
\text { [11 }\end{array}$ & $30-45$ min & $2-3$ & yes & no & nes & no & Music therapist & no \\
\hline Massage [11] & $10-30$ min & 5 & no & yes & yes & no & yes & minimal & no \\
\hline $\begin{array}{l}\text { Namaste } \\
\text { Care [16] }\end{array}$ & $8 \mathrm{hrs}$ & 7 & & no & yes & no \\
\hline
\end{tabular}

Table 1: Strategies used to improve quality of life in advanced dementia

Special environment: Namaste Care takes place in a small group setting, usually 8-10 residents and one care provider in a space that is calm and free of distractions. By being part of a group even people with advanced dementia feel the presence of others and this leads to increased communication. Soothing music is played, lights are lowered and smell of lavender permeates the room. Residents who are not in reclining chairs are placed in comfortable seats and are always in presence of a care provider. This setting decreases agitation, and increases engagement with care providers, families and other residents. Beverages are offered continuously during both morning and afternoon session, easy to swallow snacks (e.g., ice cream or pudding) are offered in the afternoon session and improved appetite was reported.

Loving touch: Loving touch approach to care is provided by a Namaste care provider or families. It includes gentle washing of the face and application of a face moisturizes with familiar scent. Arm, hand, leg and feet are also washed and moisturized. Hair is gently combed a make- up applied to ladies. Realistic dolls, dogs and cats are offered to residents who find holding them pleasurable. Often, combination of a group environment and loving touch makes some residents who were non-verbal start to speak again. Loving touch also decreases the perception of pain and makes residents less resistive to care even after leaving the Namaste Care program. This result in decreased rejection of care, preventing reactive aggression that sometimes occur, especially during bathing.

Family members and staff reported that Namaste Care improved not only lives of the residents but also their lives. Family members enjoyed more their visits, because they were able to participate in hand massage and other activities, they had better interactions with their loved ones and felt more at peace because they saw improved condition of their loved ones. They especially appreciated that the residents were better able to communicate with them. Staff reported that participation in Namaste Care increased their job satisfaction and that their job was easier because of decreased rejection of care. Another qualitative study, using data from focus groups conducted at three residential aged care facilities located in New South Wales, Australia, found two broad themes: touch by others and touch by the person. These two broad themes, with their accompanying elements, express the essential nature of mental health as a reciprocal connectedness, with reciprocal impacts on both those people with advanced dementia and their care providers [18].

Namaste Care was not yet evaluated by a randomized control trial but several cohort studies reported beneficial effects. Using QUALID scale [8], investigators found that Namaste Care increased quality of life of the residents [17]. Another study found significant decrease of severity of behavioral symptoms of dementia and their occupational disruptiveness [19] measured by the Neuropsychiatric Inventory [20]. This decrease allowed gradual discontinuation of antipsychotic and hypnotic therapy over a period of 6 months [21]. There is also some evidence that Namaste Care decreased symptoms of depression. Using Cornell Scale for Depression in Dementia [6], investigators found that before Namaste Care implementation 10 out of 11 residents had scores 6 or above while approach. This is true for Music therapy and Namaste Care. However, Music therapy may combine residents with different degrees of cognitive impairment while Namaste Care creates a small consistent group of residents who may develop attachment with each other. Pleasurable activities are provided by several strategies but some of them in a very limited manner. People with dementia need stimulation during all their waking hours and also on the weekends, therefore, the strategy should be provided as continuous activity and happen 7 days a week. This could be true for Snoezelen integrated program and this is the basis of Namaste Care. Implementation of some strategies may be hindered by the cost of special equipment and this is especially the case of Snoezellen and possibly also some music therapy. Another barrier is the need for additional or specialized staff which is not needed in Snoezelen and Namaste Care. Finally, the extensive training requirement may cause introduction of some programs impractical.

\section{References}

1. Cahill S, Diaz-Ponce AM (2011) I hate having nobody here. I'd like to know where they all are': Can qualitative research detect differences in quality of life among nursing home residents with different levels of cognitive impairment? Aging Ment Health 15: 562-572. 
2. Folstein M, Folstein S, McHugh PJ (1975) 'Mini-mental State,' a practical method for grading the cognitive state of patients for clinicians. J PsychiatrRes 12: 189-198.

3. Gruneir A, Lapane KL, Miller SC, Mor V (2008) Is dementia special care really special? A new look at an old question. J Am Geriatr Soc 56: 199-205.

4. Hoe J, Katona C, Roch B, Livingston G (2005) Use of the QoL-AD for measuring quaility of life in people with severe dementia-the LASER-AD Study. Age Aging 34: 130-135.

5. Ready RE, Ott BR, Grace J, Fernandez I (2002). Alzheimer Disease \& Associated Disorders 16: 109-115.

6. Alexopoulos GS, Abrams RC, Young RC, Shamoian CA (1988) Cornell Scale For Depression in Dementia. Biological Psychiatry 23: 271-284.

7. Ettema TP, Droes RM, de Lange J, Mellenbergh GJ, Ribbe MW (2007) QUALIDEM: Development and evaluation of a dementia specific quality of life instrument. J Geriatr Psychiatry 22: 549-556.

8. Weiner MF, Hynan LS (2015) The quality of life in late-stage dementia. J Am Med Dir Assoc 1:114-116.

9. Cohen-Mansfield J, Werner P, Marx MS (1989) An observational study of agitation in agitated nursing home residents. International Psychogeriatrics 1: 153-165.

10. Landes AM, Sperry SD, Strauss ME, Geldmacher DS (2001) Apathy in Alzheimer's disease. Journal of the American Geriatrics Society 49: 1700-1707.

11. Strom BS, Ytrehus S, Grov EK (2016) Sensory stimulation for persons with dementia: a review of the literature. J Clin Nurs 25:1805-1834.

12. Livingston G, Kelly L, Lewis-Holmes E, Baio G, Morris S, et al. (2014) A systematic review of the clinical effectiveness and cost-effectiveness of sensory, psychological and behavioural interventions for managing agitation in older adults with dementia. Health Technol Assess 18: 1-226.
13. Livingston G, Kelly L, Lewis-Holmes E, Baio G, Morris S, et al. (2014) Nonpharmacological interventions for agitation in dementia: systematic review of randomised controlled trials. British Journal of Psychiatry 205: 436-442.

14. van Weert JCM, van Dulmen AM, Spreeuwenberg PMM, Ribbe MW, Bensing J M (2005) Behavioral and Mood Effects of Snoezelen Integrated into 24-Hour Dementia Care. JAGS 53: 24-33.

15. Moyle W, Cooke ML, Beattie E, Shum DH, O'Dwyer ST, et al. (2014) Foot massage versus quiet presence on agitation and mood in people with dementia: a randomised controlled trial. Int J Nurs Stud 51: 856-864.

16. Simard J (2013) The End-of-Life Namaste Program for People with Dementia.

17. Manzar BA, Volicer L (2015) Effects of Namaste Care: Pilot Study. American Journal of Alzheimer's Disease 2: 24-37.

18. Nicholls D, Chang E, Johnson A, Edenborough M (2013) Touch, the essence of caring for people with end-stage dementia: a mental health perspective in Namaste Care. Aging \& Mental Health 17: 571-578.

19. Stacpoole M, Hockley J, Thomsell A, Simard J, Volicer L (2015) The Namaste Care programme can reduce behavioural symptoms in care home residents with advanced dementia. Int J Geriatr Psychiatry 30: 702-709.

20. Cummings JL, Mega M, Gray K, Rosenberg-Thompson S, Carusi DA, et al. (1994) The neuropsychiatric inventory: Comprehensive assessment of psychopathology in dementia. Neurology 44: 2308-2314.

21. Fullarton J, Volicer L (2013) Reductions of antipsychotic and hypnotic medications in Namaste Care. J Am Med Dir Assoc 14: 708-709.

22. Soliman A, Hirst S (2015) Using sensory activities to improve dementia care. Nursing Times 111:12-15. 